\title{
Modeling human lung infections in mice
}

\author{
Respiratory pathogens and the human immune response they elicit can now be studied in humanized mice.
}

\author{
Hergen Spits and Julien Villaudy
}

R esearch on human pathogens is often hampered by the lack of appropriate animal models that can recapitulate a normal infection cycle and pathogenesis. Many human pathogens show preferential, often exclusive, tropism for human cells and do not replicate in experimental animals. In this issue, Wahl et al. ${ }^{1}$ provide mouse models to study respiratory pathogens, which are the leading cause of death by infectious disease globally and the fourth most common cause of death overall. The mice carry both human fetal lung tissue and a humanized immune system, allowing the study of pathogen replication in human cells from the respiratory tract and of human immune responses to the pathogens in an animal model. They are especially suited to the investigation of pathogens targeting cells from the lower respiratory tract, particularly pathogens for which no relevant animal models exist, such as human cytomegalovirus (HCMV), which replicates only in humans.

Wahl et al. ${ }^{1}$ build on decades of work on developing immunodeficient mice that allow engraftment of human immune cells and tissues (Fig. 1). Several mouse strains are available that support engraftment of human umbilical cord blood, mobilized blood, bone marrow and fetal tissues such as liver and thymus ${ }^{2,3}$. Human hematopoietic stem cells are typically engrafted in strains that lack or have inactivating mutations in genes required for rearrangement of $\mathrm{T}$ cell and B cell receptor genes (e.g., recombinase activating genes (Rag) or the DNA-dependent serine/threonine protein kinase (Prkdc)) and in the $\gamma$ common chain of the interleukin (IL)-2 receptor (IL2R), which is critical for development of natural killer cells. All these strains lack mouse $\mathrm{T}$ cells, $\mathrm{B}$ cells and innate lymphoid cells, including natural killer cells. A frequently used strain, which is also used by Wahl et al. ${ }^{1}$, is a non-obese diabetic-severe combined immunodeficiency (NOD-SCID) strain with a Prkdc mutation and an IL2R $\gamma$ deficiency, called NSG mice.

These highly immunodeficient strains serve to create two types of mice with a humanized immune system. Human immune system (HIS) mice are generated

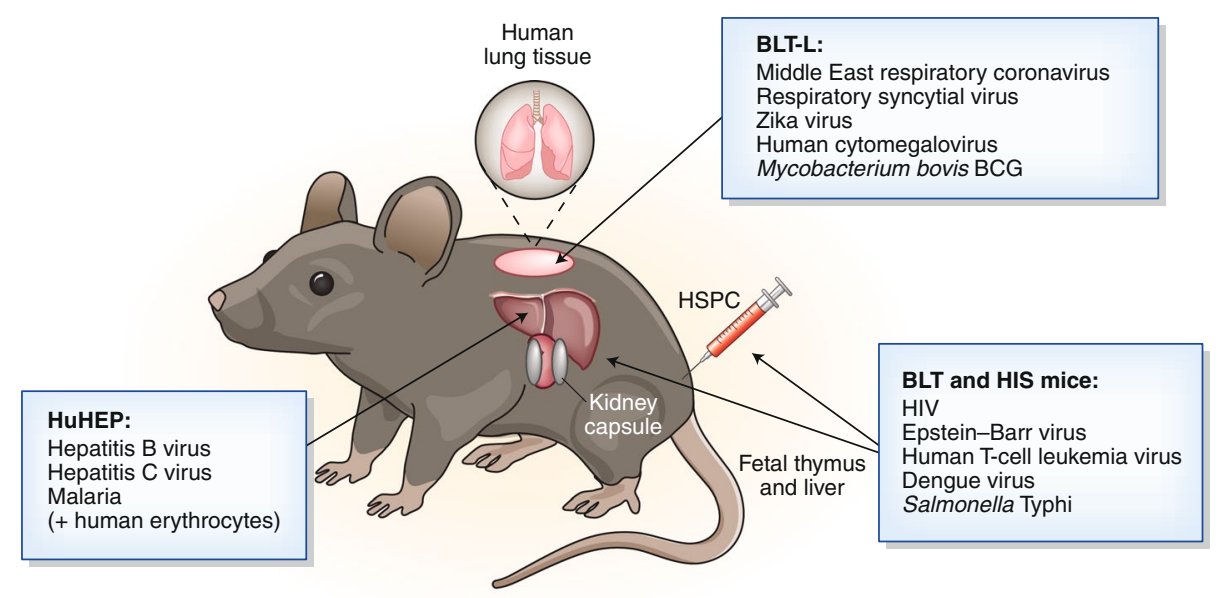

Fig. 1 | Humanized mice to study human-tropic pathogens. Various human cell types have been transplanted into immunodeficient mice to study diverse pathogens and diseases. Human immune system (HIS) mice are engrafted with hematopoietic stem cells. Bone marrow-liver-thymic (BLT) mice are engrafted with hematopoietic stem cells, fetal liver and fetal thymus from the same donor. HuHEP mice are engrafted with human liver cells to study hepatotropic viruses and, when combined with human erythrocytes, the replication cycle of the malaria parasite. BLT-L mice, now developed by Wahl et al.', are engrafted with lung cells, with or without autologous immune cells, to study respiratory viruses.

by simply injecting the mice with human hematopoietic stem cells ${ }^{4-7}$. In these mice, human $\mathrm{T}$ cells develop in the mouse thymus. Bone marrow-liver-thymic (BLT) mice, a more complex model, require transplantation of human fetal liver and thymus under the kidney capsule, followed by injection of bone marrow hematopoietic stem cells from the same donor. The presence of human liver (as a source of stem cells) and thymus in BLT mice supports more robust $\mathrm{T}$ cell development than observed in HIS mice, whereas co-injection of hematopoietic stem cells optimizes development of B cells and other hematopoietic cells.

In both HIS and BLT mice, cells from the different human hematopoietic lineages, including B cells and T cells, are present in primary and secondary lymphoid organs, as well as in peripheral blood ${ }^{2,3,9}$. Both are excellent models for studying humanspecific pathogens that target hematopoietic cells, such as human immunodeficiency virus, Epstein-Barr virus and human T-cell leukemia virus. To study pathogens that infect non-hematopoietic cells, researchers can transplant human tissues along with hematopoietic cells. For example, mice engrafted with human immune cells and human hepatocytes (HIS-HuHEP mice) ${ }^{10}$ have been used to study immune reactions against human liver trophic viruses like hepatitis B virus ${ }^{11}$.

The new study by Wahl et al. substantially extends the number of human pathogens that can be modeled in humanized mice. The authors first transplant pieces of human fetal lung subcutaneously in the back of NSG mice to create 'human lung-only' (LoM) mice. Using an elegant technology called acoustic angiography, they demonstrate the occurrence of active angiogenesis in and around the implants. Interestingly, although the lung implants are ectopic and therefore not ventilated, their structural features are highly similar to those of normal human lung, including ciliated epithelium, alveolar structures, blood vessels and cartilage. 
Wahl et al. ${ }^{1}$ show that the grafts support robust replication of clinically relevant human respiratory pathogens that have no or limited tropism to non-human cells. These pathogens-including Middle East respiratory coronavirus, Zika virus, respiratory syncytial virus and HCMVrepresent a risk for human health globally. The authors also study Mycobacterium bovis bacillus Calmette-Guerin, which is used to vaccinate against $M$. tuberculosis. Moreover, the authors measure the expression of viral genes during lytic replication of HCMV in vivo for the first time.

Because LoM mice lack an immune system, pathogen replication is uncontrolled. To mimic infection as it occurs in healthy humans, the authors continue their remarkable study by combining LoM and BLT models to create mice with both a lung transplant and an immune system, called BLT-L mice. For this, NSG mice are first transplanted with human fetal liver and thymus under the kidney capsule, followed by implantation of autologous lung tissue, and finally injection of autologous liver-derived hematopoietic stem cells.

The level of repopulation with human hematopoietic cells outside the lung transplants is as robust in BLT-L as in BLT mice. The lung grafts also have a substantial population of human immune cells; whether this population was present in the lung grafts before implantation or whether it derives from the injected hematopoietic cells is unclear, but its cell composition-notably, the relative proportions of CD4 T cells, CD8 $\mathrm{T}$ cells and tissue-resident memory $\mathrm{T}$ cellsclosely recapitulates that of hematopoietic cell populations in human lungs.
The authors provide convincing evidence that the immune response to HCMV in BLT-L mice is functional and can control viral replication. Plasma levels of pro- and anti-inflammatory cytokines including interferon- $\gamma$, IL-6, granulocyte-macrophage colony stimulating factor (GM-CSF) and chemokines such as IL- 8 and interferon- $\gamma$ induced protein 10 (IP10) were increased after the infection, indicative of an ongoing immune response. The adaptive immune response in these mice includes both antigenspecific, cytokine-producing T cells and virus-specific antibodies. HCMV-reactive immunoglobulin $\mathrm{M}$ is detected 14 days after primary infection. Class switching to HCMVspecific immunoglobulin $\mathrm{G}$ is not observed upon single exposure but is detected in nearly $75 \%$ of the mice re-exposed to HCMV.

Wahl et al. ${ }^{1}$ did not investigate whether there is affinity maturation of the HCMVspecific IgGs. If the mice support somatic hypermutation, this would represent an important advance because thus far highaffinity, class-switched, antigen-specific antibodies have rarely been produced in humanized mouse models. There are several possible reasons for this. One is that the host strains of HIS and BLT mice have only a few secondary lymph nodes owing to a lack of IL2Ry-dependent lymphoid tissue inducer cells. Another is that there is a suboptimal interaction of $\mathrm{B}$ cells and $\mathrm{T}$ cells caused by a lack of cross-reactivity on the part of critical cytokines involved in this interaction. Also, non-hematopoietic cells important for B cell maturation are of mouse origin and do not appropriately interact with maturing B cells. This may also be a reason for the lack of structured germinal centers in immunized HIS and BLT mice.
It will be important to analyze the $B$ cell response in the BLT-L mice in detail. If the mice indeed generate not only class-switched but also affinitymatured antibodies, their utility would be greatly enhanced. For instance, this would allow testing of new vaccine strategies, antiviral drugs and antibiotics. It would also be possible to generate broadly reacting, highly neutralizing antibodies against clinically relevant pathogenic viruses.

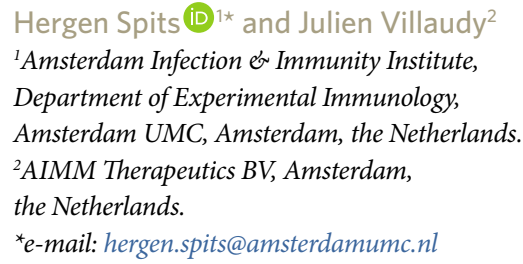

Published online: 10 September 2019

https://doi.org/10.1038/s41587-019-0269-x

References

1. Wahl, A. et al. Nat. Biotechnol. https://doi.org/10.1038/s41587019-0225-9 (2019).

2. Shultz, L. D., Brehm, M. A., Garcia-Martinez, J. V. \& Greiner, D. L. Nat. Rev. Immunol. 12, 786-798 (2012).

3. Rongvaux, A. et al. Annu. Rev. Immunol. 31, 635-674 (2013).

4. Gimeno, R. et al. Blood 104, 3886-3893 (2004)

5. Traggiai, E. et al. Science 304, 104-107 (2004).

6. Ito, M. et al. Blood 100, 3175-3182 (2002)

7. Shultz, L. D. et al. J. Immunol. 174, 6477-6489 (2005).

8. Melkus, M. W. et al. Nat. Med. 12, 1316-1322 (2006).

9. Ito, R., Takahashi, T., Katano, I. \& Ito, M. Cell. Mol. Immunol. 9, 208-214 (2012).

10. Strick-Marchand, H. et al. PLoS One 10, e0119820 (2015).

11. Dusséaux, M. et al. Gastroenterology 153, 1647-1661.e9 (2017).

Competing interests

J.V. is an employee of and stockholder in AIMM Therapeutics. H.S. owns stock in AIMM Therapeutics and consults for GlaxoSmithKline. 